\title{
Effect of Tai Chi Combined With Kinesio Taping on Posture Control of Football Players With FAl: Protocol For a Randomized Controlled Trial
}

YH LI ( $\square$ li2513436@163.com )

Beijing Sports University https://orcid.org/0000-0002-3213-5626

\section{Youhua Li}

China Wushu School, Beijing Sport University, Beijing 100084, China

\section{Study protocol}

Keywords: Functional ankle instability, Tai Chi, kinesio taping, posture control, ankle joint, balance

Posted Date: July 14th, 2021

DOl: https://doi.org/10.21203/rs.3.rs-676720/v1

License: (c) (1) This work is licensed under a Creative Commons Attribution 4.0 International License. Read Full License

Version of Record: A version of this preprint was published at Trials on February 19th, 2022. See the published version at https://doi.org/10.1186/s13063-022-06083-5. 


\section{Abstract}

Background: Functional ankle instability (FAl) of college football players is an important risk factor affecting their training and competition. Physical therapy and appropriate sports intervention can improve the stability of FAl patients. Previous studies have shown that Tai Chi (TC) and Kinesio Taping (KT) can improve the posture control ability of FAl patients. However, whether Tai Chi combined with kinesio taping effect patch can be used as an effective exercise for the rehabilitation of college football players with FAl is not yet proven.

Methods/design: $53 \mathrm{FAl}$ college football players were randomly assigned to 3 groups: TC+KT $(n=20)$; TC+KTp (Placebo Kinesio taping, KTp, placebo) $(\mathrm{n}=17) ; \mathrm{KT}(\mathrm{N}=16)$. The TC+KT group received TC and KT functional correction technical intervention, the TC+KTp group received TC and placebo KT technical intervention, and the KT group received KT functional correction technical intervention. Each of the three groups received 30 minutes each time, 3 times a week, for a total of 6 weeks of intervention training. Star Excursion Balance Test (SEBT) and UniPedal Stance Test (UST) at baseline (before), 4 weeks after intervention (middle) and 6 weeks after intervention (after) , Toe Touch Test (TTT) for evaluation.

Discussion: For the first time in this trail, the impact will be evaluated. If the results are the same as expected, they will provide evidence that Tai Chi combined with kinesio taping sticking intervention can promote the posture control of college football players with FAI.

Trial registration: Chinese Clinical Trial Registry, ChiCTR1900027253.Registered on 6 November 2019.

\section{Background}

Football is the world's most popular sport. It has the characteristics of fighting fierce, repeated kicks, rapid changes of direction, instant acceleration, instant jumping and landing. This feature makes it one of the sports with the highest incidence of sports injuries[[i]]. Much higher than basketball and handball[[ii]]. Football injuries are mainly concentrated in the lower limbs[[iii]], especially the ankle joints[[iv]][[v]], which account for about $85 \%$ of the total football injuries[[vi]]. Due to the incomplete rehabilitation or overuse of the football player's ankle joint, it is easy to cause repeated injuries. It is the highest risk of recurrence in football injuries, reaching $80 \%[[$ iii]]. Over time, functional ankle instability (FAl) will gradually develop. At present, there is no expert consensus on the mechanism of FAI. Relevant studies have shown that it is related to the loss of proprioceptive sensitivity[[viii]][[ix]], loss of strength[[x]] and weakened sense of balance after ankle joint injury[[xi]].

In order to ensure the proper training and competition of FAl university football players, effective rehabilitation is still a difficult problem to solve in a short time. At present, previous research on FAI rehabilitation mainly focuses on its proprioception[[xii]][[xiii]], strength[[xiv]][[xv]], balance training[[xvi]], strength and proprioception intervention[[xvii]], and motor sensory training[[xviii]]. To a certain extent, reduce the recurrence rate of ankle sprains. 
In addition to exercise therapy, other strategies for FAI intervention are also suggested to improve the flexibility and stability of the ankle joint. For example, KT improves the vertical jump effect of FAI[[xix]], KT improves the functional performance of female basketball players with unstable ankle joints[[xx]], and KT improves the dynamic balance of FAII[xxi]]. Studies have shown that KT can also increase muscle strength[[xxii]], enhance proprioception[[xxiii]], Improve flexibility[[xxiv]]. At the same time, some studies have reported the opposite result $[[\mathrm{xxv}]]$. At present, although most studies have proved the positive effects of $\mathrm{KT}$, no scientific consensus has been formed, and more research is needed to promote the consistency of KT's effects.

Tai Chi is a sports event with the main feature of single-leg support of the lower limbs. It has been widely used in the promotion of human health. The role of posture control is mainly manifested in improving the proprioception of the elderly[[xxvi]], balance[[xxvii]] and preventing falls[[xxviii]], knee osteoarthritis[[xxix]] In other aspects, there are few studies on Tai Chi's intervention in FAl, especially the intervention of college football players in FAI. There is insufficient evidence to prove the intervention effect of Tai Chi or combined kinesio taping effect on the posture control such as stability and flexibility of college football players with FAl patients. This trial uses a rigorously designed randomized controlled trial to evaluate the influence of Tai Chi intervention or combined intervention with KT on the posture control of football players with FAl.

\section{Methods/design \\ Study purposes}

The purpose of this trial is to evaluate the effectiveness of a 6-week Tai Chi or combined kinesio taping effect intervention on the posture control of college football players with FAl.

\section{Study design}

The participant flow for this trial is presented in Fig. 1. The present protocol follows the Standard Protocol Items: Recommendations for Interventional Trials (SPIRIT) guidelines and fulfills the SPIRIT checklist (see Additional file 1).

This article is a double-blind randomized controlled trial, recruiting 66 eligible college football players FAl patients, randomly divided into 3 groups: TC+KT group, TC+KTp group, KT group, the ratio is 1:1. Participants in the TC+KT group will receive 6 weeks of Tai Chi ( 3 times a week, 30 minutes each time) combined with kinesio taping effect patch intervention; TC+KTp group will receive 6 weeks of Tai Chi and muscle Placebo technical intervention with internal effect patch; KT group will receive 6-week kinesio taping effect intervention. Measurements will be taken at the baseline, mid-intervention period (4 weeks), and late intervention period ( 6 weeks) to check the maintenance of any intervention effects. The participant flow for this trial is presented in Fig. 1. The present protocol follows the Standard Protocol 
Items: Recommendations for Interventional Trials (SPIRIT) guidelines and fulfills the SPIRIT checklist (see Additional file 1).

\section{Sample size calculation}

The sample size is calculated by the software G*Power 3.1.9 for Windows (G*Power@ from University of Dusseldorf,Germany)[[i]]. The calculation of the sample size is regarded as the calculation of power, and its purpose is to observe whether there is a significant difference in the outcome indicators. This study consists of 3 groups and 3 measurements, the statistical power is 0.8 , the type I error probability (a) is 0.05 , the statistical analysis method is mixed-type analysis of variance, and the amount of effect obtained in the preliminary trail is 0.25 , which is calculated by the software The sample size is 54 participants in total, with 18 participants in each group. At the same time, considering the $20 \%$ dropout rate and the equivalence between the groups, the sample size is 66 participants, and each group has 22 participants.

\section{Setting and recruitment}

This study will recruit FAl patients at the Football Academy of Beijing Sport University in China through WeChat, leaflets, and Weibo. Exercise intervention was independently supervised by 3 research assistants at this university, and data collection was carried out independently by other research assistants. Due to the impact of the new crown epidemic, this study will officially start recruiting in September 2021.

\section{Participant eligibility}

The inclusion criteria of FAl are determined according to Delahunt (2010)[[i]]. The inclusion criteria are: (1) Repeated ankle sprains 2 times or more in the last year; (2) The most recent ankle sprain distance study> 1 month, in functional The ankle joint feels out of control during activities; (3) Ankle Function Evaluation Questionnaire (AJ-FAT) score $\leq 26$ points; (4) Ankle joint anterior drawer test and talus tilt test results are negative; (5) No fracture or lower limb Medical history such as surgery; (6) Voluntary and signed informed consent. The exclusion criteria are: (1) Ankle sprain time < 1 month; (2) Ankle pain at rest; (3) Allergy to KT; (4) Medical contraindications for physical activity (such as cardiovascular disease); (5) The intervention cannot be persisted due to personal or other reasons. All subjects voluntarily and signed written informed consent before the intervention, and were explained by the researchers on the research process, and strictly followed the ethical standards of the Declaration of Helsinki.

\section{Randomization, allocation, and blinding}

Random grouping is carried out by individual researchers. The generation of random sequence and random list are carried out by individual researchers through SPSS (IBM. Version 20.0) software program. 
This is done on subjects who have signed the "Informed Consent". Make sure the group is hidden. Participants were randomly divided into 3 groups: TC+KT $(n=20)$, Tai Chi combined with kinesio taping patch intervention; TC+KTp ( $\mathrm{n}=21)$, Tai Chi and kinesio taping patch placebo technical intervention $(n=21)$, kinesio taping patch intervention was performed alone.

In this trail, the assessments and treatments were performed by difffferent therapists. The evaluator was blinded to the subjects'assignment. All the intervention procedures were performed by the same physiotherapist who had experience in the fifield of sports physical therapy. Both the physiotherapist and the participants were blinded to the purpose of the study. In addition, a difffferent researcher, blinded to the object of the study, carried out the data analysis.

\section{Intervention}

\subsubsection{Intervention plan}

The three different interventions lasted for 6 weeks. During this period, they normally participated in the usual football courses ((ie 2 times a week, 90 minutes each time, 1 game on weekends). Subjects in the $\mathrm{TC}+\mathrm{KT}$ and $\mathrm{KT}$ groups had both ankles KT functional correction taping technology was used. The KTp+ TC group used placebo technology (tension-free) KT. The TC training of subjects in the TC+ KT and KTp+ TC groups focused on lower limb posture control.

\section{kinesio taping}

The functional correction technique of kinesio taping effect sticking is a kind of kinesio taping effect sticking technology[[ii]]. The width of the patch is $5 \mathrm{~cm}$, the thickness is $0.05 \mathrm{~cm}$, with $70 \%$ tension, and it is pasted on both ankles[[iii]]. The method of sticking is as follows: the subject lies on his back with the toes facing up, and the patch goes across the metatarsal bones from the outside of the instep to the inside without applying tension. Then reach the lateral malleolus through the sole of the foot with $70 \%$ tension, traverse the lateral ligament of the ankle joint, extend up to the end of the gastrocnemius tendon, and stick the remaining part in the middle of the gastrocnemius without tension. The calculation of the tension percentage is based on the length of the KT spread on the paper as the base point $(0 \%)$, stretch the patch with the maximum tension, and measure its length (100\%). Therefore, the tension required for $\mathrm{KT}$ in this study is $70 \%$, that is, the difference between the maximum usable tension required by the patch and the base point length is $70 \%[[i v]]$. After the taping was completed, the subjects were asked whether there was pain, and a half squat was performed to compare the pain. If there is no pain, the tape is completed.

Ankle joint injury is usually a strain of the lateral ligament. Therefore, the purpose of KT functional correction technology is to make the ankle joint valgus and prevent varus, so as to prevent ankle joint 
sports injuries[[v]].

\section{Placebo Kinesio Taping}

Thepasting of the patch is the same as before, but the tension of the patch is $0 \%$. The patch is only attached to the subject's ankle without any stretching. Both functional correction tape and placebo tape were applied to both ankles of the subjects, and tape was taped once every 5 days[[vi]]. If the subject discovers any skin allergies when changing $\mathrm{KT}$, they will be excluded from the study.

\section{Tai Chi}

The intervention actions of Tai Chi are selected from the 24 styles of Tai Chi[[vii]]: Knee-knee and stance, inverted brachial, clouded hand, left-right and lower-independence (including footsteps, piercing palms and golden rooster independence), and jade girl shuttle. 5 movements, knee and stance, inverted brachial, clouded hand, left and right golden rooster independent, and jade girl shuttle, including single foot support, single foot left support, single foot right support, left support, right heel contact with the ground, right support, left heel contact with the ground, Left support, right toe touches the ground, right support, left toe touches the ground, a total of 7 support methods and different movement directions (front and back, left and right, up and down, front left, front right). These actions are mainly to control the balance and posture of the lower limbs of the body. In addition to the normal class and training, the subjects intervened in the reference guide[[viii]] and adjusted according to the characteristics of the subjects, and practiced 3 times a week, each for 30 minutes. The intervention is divided into two stages: learning in the first 2 weeks and improvement in the next 4 weeks. Each exercise in the first 2 weeks includes 5 minutes of warm-up, 20 minutes of learning new movements, and 5 minutes of stretching. The last 4 weeks include 5 minutes of warm-up, 20 minutes of repetitive exercises, and 5 minutes of stretching.

\section{Outcome assessment test indicators}

After the subjects agreed to participate in the intervention, a preliminary assessment was made. Tests were performed before the intervention (baseline), four weeks later (mid-term), and six weeks (end of intervention). The tested variables include: (1) dynamic balance; (2) static balance; (3) flexibility.

\section{Primary outcome}

Dynamic balance: Dynamic balance is evaluated using the Star Excursion Balance Test (SEBT)[[ix]]. SEBT is a comprehensive quality including strength, flexibility, and coordination. In the test, the subject is required to stand on one foot, maintain physical balance and use the affected leg as the support. The non-supporting leg faces the front of the supporting leg. Extend as far as possible in the posterior-inward direction and posterior-outward direction, retract and close the supporting leg, and then start the next 
extension. During the test, the subject's supporting leg moved or the non-supported leg touched the ground were considered failures. The farthest point of the unsupported leg from the starting point to the extension direction is the farthest distance $(\mathrm{cm})$ reached by the subject, which is also the target data for all tests in this study. Before the formal test, the subjects performed 4 test exercises. The subjects measured 3 times in each direction, and rested for 20 seconds between each time. The average of the 3 test data was the subject's final test score. SEBT has been proven to be an effective method for evaluating ankle stability[[x]], and it has good internal reliability $(\mathrm{ICC}=0.86-0.94)$ and retest reliability $(\mathrm{ICC}=0.89-0.93)[[\mathrm{xi}]]$.

Static balance: Static balance is assessed by the UniPedal Stance Test (UST) with closed eyes. The subjects were asked to stand barefoot, eyes closed, arms crossed on the chest, legs close together, and toes forward. When the subject heard the start command, the subject closed his eyes and chose the affected foot as the supporting leg to stand, and the other leg was bent and raised to the height of the knee joint of the supporting leg until the subject lost his balance. Calculate the effective time for the subject to stand on one leg with a stopwatch, and start timing when the start command is issued. The time will stop when one of the following situations occurs: (1) Arms are not crossed over the chest; (2) Use a non-supporting leg to touch the ground or stay away from the supporting leg; (3) The supporting leg is displaced; (4) Eyes are opened; (5) ) The standing time has reached 45 seconds. Each subject performed 3 repetitive tests with a 20-second break between each test. The average of the 3 tests was used as the subject's final test score and used for statistical analysis. The closed-eye single-foot test has been proved by related studies to have high score reliability $(I C C=0.998)[[x i i]]$.

\section{Secondary outcome}

Flexibility: Flexibility is evaluated by the Toe Touch Test (TTT). The subject stood on the wooden box with his feet aligned, toes facing forward, straight legs bent at the hips, and tried his best to touch the toes with his hands. The knee joints could not bend while the body was bent down. Subjects are required to do their best to complete the test without bending or pain in the knee joint during the test. The value of the ruler touched by the hand is the distance $(\mathrm{cm})$ reached by the subject. Each subject performed 3 repetitive tests with a 20-second break between each test. The average of the 3 tests was used as the subject's final test score and used for statistical analysis. The standing flexion test has been proven to have good testretest reliability $(I C C=0.89)[[x i i i]]$.

\section{Safety measurements}

Any unexpected adverse events that occurred during the 6-week intervention period will be reported to the research assistant, and the causal relationship between y and Tai Chi exercises and kinesio taping effects will be evaluated. If a serious sports injury or other adverse event occurs, the research assistant will immediately report to the project leader and the Sports Science Ethics Committee of Beijing Sport University; they will decide whether the participant needs to withdraw from the study. 


\section{Data collection}

The demographic information of the subjects will be collected during the recruitment process. The data of the primary and secondary results will be collected by specialized result assessors at baseline, 4 weeks of intervention, and 6 weeks of intervention. All result assessors conduct standardized training on test methods before intervention to ensure that all subjects have equal test conditions.

In order to ensure the attendance rate of the participants and provide more complete data results, we will provide all participants with free Tai Chi champion teaching services and a reward of 100 yuan. It will be distributed through WeChat after 6 weeks of intervention.

\section{Data management}

The main and secondary results of the test will be recorded through the case report form (p-CRF), and the paper version of the data will be processed electronically through the free data management software EpiData Manager in a timely manner. Two result evaluators separately reviewed and confirmed the data, and converted it into a format that can be used for statistical analysis.

\section{Statistical analysis}

Statistical analysis was performed through the statistical package for social sciences software (The Statistical Package for Social Sciences, SPSS 23, SPSS Inc., Chicago, IL, USA). The normal distribution of variables is carried out by ShaPiro-Wilk. Descriptive statistical analysis is described in the form of mean \pm standard deviation. Through mixed two-factor multivariate analysis of variance, the effects of intervention groups (such as TC $+\mathrm{KT}, \mathrm{KTp}+\mathrm{TC}, \mathrm{KT}$ ) and time (such as before, during, and after intervention) were analyzed by reasoning. Multiple comparisons were made through post-hoc analysis and bonferroni correction for the variables of the variance analysis significance results. According to Cohen's method, the effect amount is calculated. This method divides the effect amount into large (0.8), medium (0.50-0.79), and small (0.20-0.49) [[xiv]]. The alpha level for all tests is set to 0.05. A single-factor multivariate analysis of variance was used to compare the subjects' baseline age, height, weight, and leg length data to explore whether there is homogeneity between the groups.

\section{Ethics}

The conduct of this research will comply with the principles of the Declaration of Helsinki and relevant ethical guidelines, including informed consent and confidentiality and data storage. The ethics was approved by the Ethics Committee of Beijing Sport University Sports Science trail (Approval Number 2019097H). All participants will be fully informed of the trial situation and signed an informed consent form before participating. 


\section{Monitoring}

Tai Chi is an aerobic exercise with low risk. This study is not expected to cause any potential harm. Therefore, there will be no data monitoring committee, temporary analysis or stopping rules. we do not anticipate any potential harms. Therefore, there will be no Data Monitoring Committee, interim analyses, or stopping rules.

\section{Dissemination}

The research protocol has been registered and can be viewed on the China Trial Registration website (registered at ChiCTR.org, with the identifier ChiCTR1900027253). The research results will be disseminated to all participants, researchers, healthcare providers and sponsors through research summary documents, courses, presentations and the Internet. The research will also be published in scientific journals and presented at conferences, targeting a wide range of groups.

The results will be disseminated to all participants, researchers, healthcare providers, and sponsors through study summary documents, courses, presentations, and the Internet. This study will also be published in scientific journals and be presented at conferences to target a wide range of groups .

\section{Discussion}

Tai Chi is a kind of traditional sports that is unique to our country that supports both body and mind, and both internal and external training. In recent years,

Tai Chi has been clearly recommended as one of the exercise therapies by major clinical guidelines[[i]][iii]] [[iii]]. Especially after the intervention research results of Tai Chi were published in the world-renowned "New Eng-land Journal of Medicine"[[iv]][[v]], it has been confirmed that Tai Chi is more effective. The role of preventive medicine and public health. Chung $\mathrm{PH}$ et al. [[vi]]divided 48 healthy youths into a Tai Chi combined vibration training group, a Tai Chi training group, and a control group. They conducted 8 weeks of intervention ( 3 times a week, 30 minutes each time), and found that Tai Chi training can significantly improve Lower limb strength and balance control, balance control is significantly improved. Its efficacy has also been proven in other studies of Tai Chi intervention[[vii]][[viii]]. The positive rehabilitation effect of Tai Chi on FAl patients has also been proved by research[[ix]]. Therefore, Tai Chi may also have a positive effect on the posture control ability of college football players with FAI.

The KT functional correction technique used in this study can prevent the ankle joint from being stretched beyond the normal range of motion, thereby preventing sports injuries[[x]]. The results of previous studies show that compared with the acute effects of KT, long-term interventions (4 weeks) for college football players' ankle posture control (dynamic balance, static balance) have no significant effect[[xi]]. The results of other studies are also consistent, indicating that KT There is no significant effect on the dynamic balance of SEBT[[xii]]. In terms of flexibility, KT is significantly better than PNF after 
intervention[[xiii]]. At the same time, most studies on the short-term or acute effects of KT have also shown significant results[[xiv]][[xv]].

The rehabilitation effect of Tai Chi combined with KT on FAl patients has not been found yet. The results of a meta-analysis emphasize that the best rehabilitation training methods other than non-surgical FAI have not yet been determined[[xvi]]. In this trial, we will observe the effect of 6-week Tai Chi or combined kinesio taping effect on the posture control of college football players with FAl patients. Strict research design random allocation and blinded evaluation and statistical analysis will be used to reduce bias. It is expected that this test will produce reliable results.

This study has certain potential limitations. As training time conflicts with other commitments, bad weather and other emergencies, the participants in the Tai Chi exercise group adhere to the Tai Chi exercise program may be another issue that affects the research.

\section{Trial status}

The test is in progress. Recruitment starts on September 1, 2021, and ends on October 30, 2021. The test procedure is expected to be completed by the end of December 2021.

\section{Abbreviations}

KT: Kinesiotaping; TC囚Tai Chi; KTp: Placebo Kinesiotaping; UST: Unipedal Stance Test; SEBT: Star Excursion Balance Test; TTT: Toe Touch Test;FAl:Functional Ankle Instability;

\section{Declarations}

\section{Acknowledgements}

The authors are grateful to the research assistants and coordinators at all participating sites.

\section{Authors' contributions}

LYK was involved in the conception and design of the research. LYH obtained ethics approval. LYH drafted the manuscript. All authors edited and revised the manuscript. All authors approved the final version of the manuscript.

\section{Funding}


This research is funded by the Key Project commissioned by the Wushu Research Institute of the State Sports General Administration (WSH2018A005).

\section{Availability of data and materials}

Data for the study can be made available upon request. Interested researchers should contact Dr. Li at li2513436@163.com.

\section{Ethics approval and consent to participate}

The study protocol has been approved by the Sports Science trailal Ethics Committee of Beijing Sport University (approval number 2019097H). Signed consent forms will be obtained from all participants prior to their participation in the trial.

\section{Consent for publication}

Not applicable.

\section{Competing interests}

The authors declare that they have no competing interests.

\section{References}

1. [[1]] HÄGGLUND M, WALDÉN M, EKSTRAND J., 2009.Injuries among male and female elite football Players [J]. Scandinavian journal of medicine \& science in sports, 19 (6):819-827.

2. [[1]] NOYA J S, GÓMEZ-CARMONA P M, MOLINER-URDIALES D, et al., 2014. An examination of injuries in sPanish Professional soccer league [J].The Journal of sports medicine and Physical fitness, 54 (6):765-771.

3. [[1]] Dvorak, J., \& Junge, A. (2000). Football injuries and Physical symptoms. A review of the literature [J]. The American Journal of Sports Medicine, 28 (5SuPPI), 3-9.

4. [[1]] Jain T K, Wauneka C N, Liu W., 2014. The effect of balance training on ankle proprioception in patients with functional ankle instability [J]. Journal of Foot and Ankle Research, 7(1Supplement):A37.

5. [[1]] Walls, R. J., Ross, K. A., Fraser, E. J., Hodgkins, C. W., Smyth, N. A., Egan, C. J., Kennedy, J. G., 2016. Football injuries of the ankle: A review of injury mechanisms, diagnosis and management $[\mathrm{J}]$. World Journal of Orthopedics, 7(1): 8-19. 
6. [[1]] BeBeynnon, B. D., MurPhy, D. F., \& Alosa, D. M., 2002. Predictive factors for lateral ankle sprains: A literature review [J]. Journal of Athletic Training, 37(4), 376-380

7. [[1]] HALE S A, HERTEL J, 2005. Reliability and sensitivity of the foot and ankle disability index in subjects with chronic ankle instability [J]. J Athl Training, 40(1):35-40.

8. [[1]] AYALA F, CALDERÓN-LÓPEZ A, DELGADO-GOSÁLBEZ J C, et al., 2017. Acute effects of three neuromuscular warm- up strategies on several Physical Performance measures in football Players [J]. Plos one, 12(1):e0169660.

9. [[1]] STEIB S, PFEIFER K, 2015. Sensorimotor deficits in functional ankle instability [J]. Z fur Orthopadie und Unfallchirurgie,153(3):253-258.

10. [[1]] GROOMS D R, PALMER T, ONATE J A, et al., 2013. Soccer-specific warm up and lower extremity injury rates in collegiate male soccer players[J].Journal of athletic training, 48(6):782-789.

11. [[1]] RÖSSLER R, DONATH L, BIZZINI M, et al., 2016. A new injury prevention programme for children's football - FIFA 11+ kids - can improve motor Performance: a cluster- randomised controlled trial[J].Journal of sports sciences, 34(6):549-556.

12. [[1]] AMRINDER S, DEEPINDER S,SINGH S J,2012. Effect of proprioceptive exercises on balance and center of pressure in athletes with functional ankle instability [J]. Sports Med J/Medicine Sportivâ, 8(3):1927-1933.

13. [[1]] ELSHEMY S A, BATTECHA K H, 2013. Kinesio taping versus proprioceptive training on dynamic Position sense of the ankle and eversion to inversion strength ratios in children with functional ankle instability [J]. Medical J Cairo University, 81(2):61-68.

14. [[1]] HANCI E, SEKIR U, GUR H, et al., 2016. Eccentric training improves ankle evertor and dorsiflexor strength and proprioception in functionally unstable ankles [J]. Am J Phys Med Rehabil, 95(6):448458.

15. [[1]] Ha S Y , Han J H , Sung Y H . Effects of ankle strengthening exercise program on an unstable supporting surface on proprioception and balance in adults with functional ankle instability[J]. Journal of Exercise Rehabilitation, 2018, 14(2):301-305.

16. [[1]] Cruz-Diaz, D., Lomas-Vega, R., Osuna-Perez, M. C., Contreras, F. H., \&Martinez-Amat, A., 2015. Effects of 6 weeks of balance training on chronic ankle instability in athletes: A randomized controlled trial [J]. International Journal of Sports Medicine, 36(9):754-760.

17. [[1]] KIM K J., 2013. Impact of combined muscle strength and proprioceptive exercises on functional ankle instability [J]. J Int Acad phys Therapy Res, 4(2):600-604.

18. [[1]] AHMED A F,2010. Effect of sensorimotor training on joint proprioception and isokinetic strength ratios in subjects with unilateral functional ankle instability [J]. Bull Faculty Phys Therapy, 15(2):2534.

19. [[1]] Ho Y H , Lin C F , Chang $C H$, et al., 2015. Effect of ankle kinesio taping on vertical jump with runup and countermovement jump in athletes with ankle functional instability [J]. Journal of Physical Therapy Science, 27(7):2087-2090. 
20. [[1]] Kilic B B, Yildiz S, Sahinkaya T, et al., 2017. The Effects of Kinesio Tape Application on Functional Performance Measurements in Young Female Basketball Players with Chronic Ankle Instability [J]. (5):206.

21. [[1]] Kwon $₫$ Marie Pickerl|『Christine Clement., 2015.s of Ankle Kinesio tape Intervention on Dynamic Balance in prticipnts with Functional Ankle Instability[C].Medicine \& Science in Spots \& Exercise, 47(5S):128-129.

22. [[1]] Zhang, S., Fu, W., Pan, J., Wang, L., Xia, R., \& Liu, Y., 2016. Acute effects of Kinesio taping on muscle strength and fatigue in the forearm of tennis Players [J]. Journal of Science and Medicine in Sport / Sports Medicine Australia, 19(6):459-464.

23. [[1]] Magalhaes, I., Bottaro, M., Freitas, J. R., Carmo, J., Matheus, J. P., \& Carregaro, R. L., 2016. Prolonged use of KinesiotaPing does not enhance functional Performance and joint ProPriocePtion in healthy young males: Randomized controlled trial [J]. Brazilian Journal of Physical TheraPy, 20(3), 213-222.

24. [[1]] Chen, C. H., Huang, T. S., Chai, H. M., Jan, M. H., \& Lin, J. J., 2013. Two stretching treatments for the hamstrings: Proprioceptive neuromuscular facilitation versus Kinesio Taping [J]. Journal of Sport Rehabilitation, 22(1):59-66.

25. [[1]] de Almeida Lins, C. A., Neto, F. L., de Amorim, A. B. C., de Brito Macedo, L., \& Brasileiro, J. S., 2013. Kinesio Taping $\circledast$ does not alter neuromuscular performance of femoral quadriceps or lower limb function in healthy subjects: Randomized, blind, controlled, clinical trial [J]. Manual Therapy, 18(1):41-45.

26. [[1]] Xu, D., 2004.Effect of tai chi exercise on proprioception of ankle and knee joints in old People [J]. British Journal of Sports Medicine, 38(1):50-54.

27. [[1]] Li F, Harmer P, Fisher $K$ J, et al., 2005.i and fall reductions in older adults: a randomized controlled trial [J]. Journals of erontology, 60(2):187.

28. [[1]] Fong S M, Ng G Y., 2006. The Effects on Sensorimotor Performance and Balance with Tai Chi Training [J]. Archives of Physical Medicine \& Rehabilitation, 87(1):0-87.

29. [[1]] Jun-Hong $Y$, Wan-Jie G , Jian S , et al. Efficacy of Tai Chi on Pain, Stiffness and Function in Patients with Osteoarthritis: A Meta-Analysis[J]. Plos ONE, 2013, 8(4):e61672.

30. [[1]] Faul, F., Erdfelder, E., Lang, A.-G., \& Buchner, A., 2007. G* Power 3: A flexible statistical Power analysis Program for the social, behavioral, and biomedical sciences [J]. Behavior Research Methods, 39(2), 175-191.

31. [[1]] Delahunt E,Coughlan GF,Caulfield B,et al., 2010. Inclusion criteria when investigating insufficiencies in chronic ankle instability[J]. Med Sci SPort Exer, 42(11):2106-2121

32. [[1]] Kase K, Wallis J, Kase T., 2012. Clinical theraPeutic applications of the kinesio taping method [M]. Kinesio Taping Asso.

33. [[1]] Gonzalez-Iglesias, J., Fernandez-de-Las-Penas, C., Cleland, J. A., Huijbregts, P., \& Del Rosario Gutierrez-Vega, M., 2009. Short-term effects of cervical Kinesio Taping on Pain and cervical range of 
motion in Patients with acute whiplash injury: A randomized clinical trial [J]. The Journal of Orthopaedic and Sports Physical Therapy, 39(7), 515-521.

34. [[1]] Bravi, R., Cohen, E. J., Quarta, E., Martinelli, A., \& Minciacchi, D., 2016. Effect of direction and tension of Kinesio Taping application on sensorimotor coordination. [J]. International Journal of Sports Medicine, 37(11): 909-914.

35. [[1]] Konradsen, L., Voigt, M., \& Hojsgaard, C., 1997. Ankle inversion injuries. The role of the dynamic defense mechanism [J]. The American Journal of Sports Medicine, 25(1):54-58.

36. [[1]] Dawood, R. S., Kattabei, O. M., Nasef, S. A., Battarjee, K. A., \& Abdelraouf, O. R., 2013. Effectiveness of Kinesio TaPing versus cervical traction on mechanical neck dysfunction [J]. International Journal of Rehabilitation Research, 2(2):1-8.

37. [[1]] Wu Dong, 2001.24-style Taijiquan introduction and improvement[M]. Shanxi Science and Technology Press.『In Chinese区.

38. [[1]] Zhu, Q., Huang, L., Xie, W., Lin, W., Zhang, Y., \& Min, F., et al, 2016. Effects of tai ji quan training on gait kinematics in older Chinese women with knee osteoarthritis: a randomized controlled trial [J]. Journal of Sport and Health Science, 5(3), 297-303.

39. [[1]] Kinzey, S. J., \& Armstrong, C. W., 1998. The reliability of the star-excursion test in assessing dynamic balance [J]. The Journal of Orthopaedic and Sports Physical Therapy, 27(5), 356-360.

40. [[1]] Olmsted L C, Carcia C R, Hertel J, et al., 2003. Efficacy of the Star Excursion Balance Tests in Detecting Reach Deficits in Subjects with Chronic Ankle Instability [J]. Journal of athletic training, 37(4):501-506.

41. [[1]] Plisky, P. J., Rauh, M. J., Kaminski, T. W., \& Underwood, F. B., 2006. Star excursion balance test as a Predictor of lower extremity injury in high school basketball Players [J]. The Journal of Orthopaedic and Sports Physical Therapy, 36(12): 911-919.

42. [[1]] Springer, B. A., Marin, R., Cyhan, T., Roberts, H., \& Gill, N. W., 2007. Normative values for the unipedal stance test with eyes open and closed [J]. Journal of Geriatric Physical Therapy, 30(1):815.

43. [[1]] Ayala, F., Sainz de Baranda, P., De Ste Croix, M., \& Santonja, F., 2012. Absolute reliability of five clinical tests for assessing hamstring flexibility in Professional futsal Players [J]. Journal of Science and Medicine in SPort / SPorts Medicine Australia, 15(2):142-147.

44. [[1]] Cohen, J., 2013. Statistical Power analysis for the behavioral sciences [M]. Routledge: Academic Press.

45. [[1]] Qaseem A, Wilt TJ, McLean RM, et al. Noninvasive treatments for acute, subacute, and chronic low back pain:a clinical practice guideline from the American College of Physicians[J]. Annals of Internal Medicine, 2017, 166(7):514-530.

46. [[1]] National GCUK. Low Back Pain and Sciatica in Over 16s:Assessment and Management[M]. National Institute for Health and Care Excellence(UK), 2016.

47. [[1]] Stochkendahl MJ, Kjaer P, Hartvigsen J, et al. National Clinical Guidelines for non-surgical treatment of patients with recent onset low back pain or lumbar radiculopathy[J]. European Spine 
Journal, 2018,27(1):60-75.

48. [[1]] WANG C,SCHMID C H,RONES R.A randomized trial of Tai Chi for fibromyalgia[J]. New England Journal of Medicine,2010,363(8):743-754.

49. [[1]] LI F,HARMER P,FITZGERALD K.Tai Chi and postural stability in patients with Parkinson's disease[J]. New England Journal of Medicine,2012,366(6):511-519.

50. [[1]] Chung PH, Lin GL, Liu C, et al. The effects of Tai Chi Chuan combined with vibration training on balance control and lower extremity muscle power[J]. Journal of Sports Science\&Medicine, 2013,12(1):19.

51. [[1]] Hallisy KM.Tai Chi beyond Balance and fall prevention:health benefits and its potential role in combatting social isolation in the aging population [J].Curr Geriatr Rep,2018;7(3):1-12.

52. [[1]] Chen EW,Fu AS,Chan KM,et al.The effects of Tai Chi on the balance control of elderly persons with visual impairment:a randomized clinical trial [J].Age Ageing,2012;41(2):254-9.

53. [[1]] D Cruz-Díaz, Kim K M , Hita-Contreras $F$, et al. Effects of a Twelve-Week Tai Chi intervention in Patients With Chronic Ankle Instability: A Randomized Controlled Trial[J]. Journal of sport rehabilitation, 2019:1-22.

54. [[1]] Kase, K., Wallis, J., \& Kase, T., 2003. Clinical therapeutic applications of the kinesio taping method (2nd ed.) [M]. Tokyo, JaPan: Ken Ikai Co. Ltd.

55. [[1]] Marta Inglés, Pilar Serra-Añó, Àlex Giménez Méndez, Manuel Zarzoso, Marta Aguilar-Rodríguez, Luis Suso-Martí, Ferran Cuenca-Martínez \& Gemma V. EsPí-LóPez , 2019. Effect of Kinesio TaPing and balance exercises on Postural control in amateur soccer Players: A randomised control trial [J]. Journal of Sports Sciences, 37(24):2853-2862

56. [[1]] Kodesh, E., \& Dar, G., 2015. The effect of kinesio tape on dynamic balance following muscle fatigue in individuals with chronic ankle instability [J]. Research in Sports Medicine (print), 23(4), 367-378.

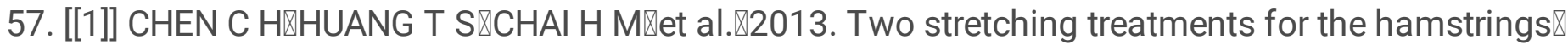
Proprioceptive neuromuscular facilitation versus Kinesio Taping[J]. J Sport Rehabil『22邓1囚:59-66.

58. [[1]] Chang, H.-Y., Chou, K.-Y., Lin, -J.-J., Lin, C.-F., \& Wang, C.-H., 2010. Immediate effect of forearm Kinesio taping on maximal grip strength and force sense in healthy collegiate athletes [J]. Physical Therapy in Sport, 11(4):122-127.

59. [[1]] de Almeida Lins, C. A., Neto, F. L., de Amorim, A. B. C., de Brito Macedo, L., \& Brasileiro, J. S., 2013. Kinesio Taping ${ }^{\circledR}$ does not alter neuromuscular performance of femoral quadriceps or lower limb function in healthy subjects: Randomized, blind, controlled, clinical trial [J]. Manual Therapy, 18(1):41-45.

60. [[1]] Kosik K B, Mccann R S, Terada M, et al., 2017.peutic interventions for improving self-reported unction in patients with chronic ankle instability: a systematic review [J]. British Journal of Sports Medicine, 51(2):105-112.

\section{Figures}




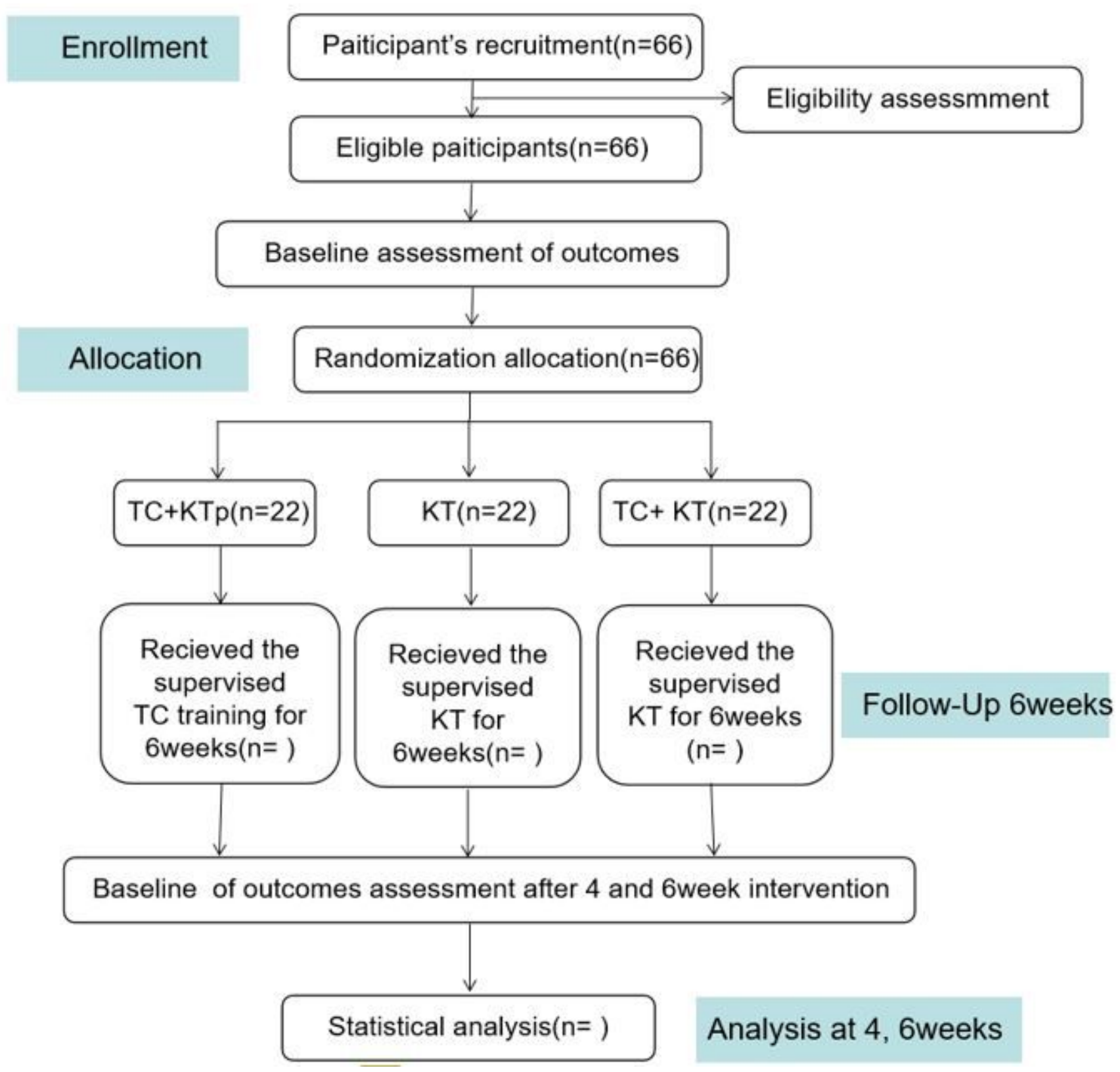

Figure 1

Proposed participant flow TC+ KT : Kinesio Taping + Tai Chi; TC+KTp: Placebo Kinesio Taping+ Tai Chi; KT:Kinesio Taping.

\section{Supplementary Files}

This is a list of supplementary files associated with this preprint. Click to download.

- PRISMA2013checklist.doc 